\title{
Thoraxtrauma: Von der präklinischen Versorgung bis in den Schockraum
}

Timo Keinert, Tobias Frech, Albrecht Henn-Beilharz
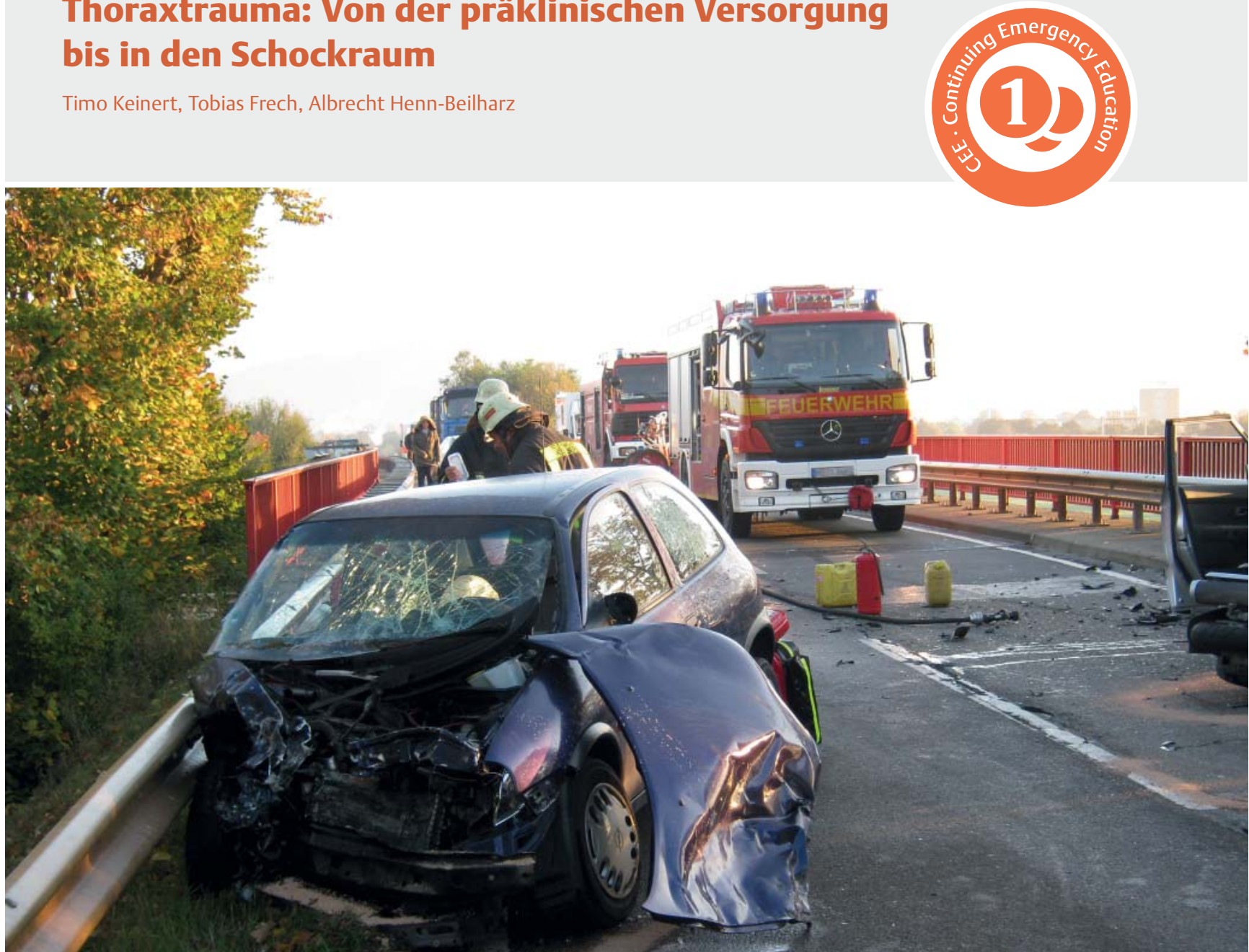

Quelle: Erik Popp/Thieme Verlagsgruppe Erik Popp

Thoraxtraumata stellen das Personal des Rettungsdienstes vor große Herausforderungen. Aufgrund pathophysiologischer Veränderungen und besonderer Dynamik haben diese Verletzungen große Relevanz und bedrohen den Patienten vital. Zeitkritische Diagnostik und Therapie stehen im Vordergrund und müssen dem präklinischen Notfallteam geläufig sein.

$\begin{array}{ll}\text { ABKÜRZUNGEN } \\ \text { AF } & \text { Atemfrequenz } \\ \text { BiPAP } & \text { Biphasic Positive Airway Pressure } \\ \text { CPAP } & \text { Continuous Positive Airway Pressure } \\ \text { DGU } & \text { Deutsche Gesellschaft für Unfallchirurgie } \\ \text { ERC } & \text { European Resuscitation Council } \\ \text { FAST } & \text { Focused Assessment with Sonography for } \\ & \text { Trauma } \\ \text { GCS } & \text { Glasgow Coma Score } \\ \text { HWS } & \text { Halswirbelsäule } \\ \text { ICR } & \text { Interkostalraum } \\ \text { NIV } & \text { nichtinvasive Beatmung }\end{array}$

PHTLS Prehospital Trauma Life Support

$\mathrm{S}_{\mathrm{p}} \mathrm{O}_{2} \quad$ pulsoxymetrisch gemessene Sauerstoffsättigung

\section{Einleitung}

Thorakale Traumata sind eine häufige Ursache für tödliche Verletzungen. Verletzungen des Brustkorbs mit den darin enthaltenen Organen (Lunge, Herz, große Gefäße) sind für das Outcome des Patienten von hoher Bedeutung, da prinzipiell jede Struktur betroffen sein kann 


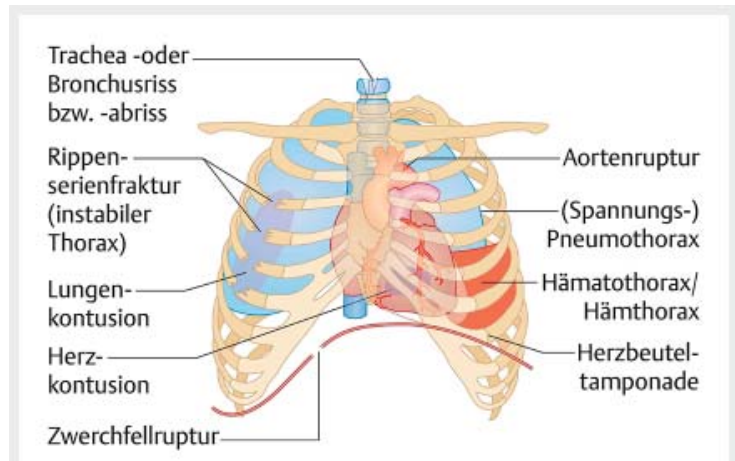

- Abb. 1 Thoraxverletzungen. Quelle: Passlick B, Stremmel W, Kroegel C. Grundlagen. In: Kroegel C, Costabel U, Hrsg. Klinische Pneumologie. 1. Auflage. Thieme; 2013. doi:10.1055/b-002-57146.

( $\triangleright$ Abb. 1). Grundlegend unterschieden werden stumpfe von penetrierenden Verletzungen. Über 90 \% sind stumpfe Thoraxtraumata, während penetrierende Verletzungen eher selten auftreten [1]. In anderen Ländern ist die Inzidenz penetrierender Verletzungen (Stich- und Schussverletzungen) deutlich höher. Möglicherweise wird sich dieses Bild in Zukunft infolge der Terroranschläge verschieben.

Daneben treffen wir zunehmend auf ältere Patienten mit Begleiterkrankungen. Bei diesen Patienten schwinden die physiologischen Reserven, Symptome sind oft verschleiert, und eine Dauermedikation (z. B. Antikoagulation) erschwert die Behandlung.

\section{Stumpfes Thoraxtrauma}

\section{Unfallmechanismus}

Bei stumpfen Traumata ist der Unfallmechanismus relevant. Ein Lenkradanpralltrauma nähert das Sternum, die Rippen und die Wirbelsäule einander an. Es kommt zur Kontusion von Lungen und Herz. Ein seitlicher Aufprall wirkt sich auf Rippen, Lunge und die großen intrathorakalen Gefäße aus. Ein Sturz aus großer Höhe bewirkt eine axiale Stauchung (s. Übersicht). Die Folgen sind nicht nur Verletzungen der Extremitäten, sondern auch des Achsenskeletts und der intrathorakalen Organe.

\section{ÜBERSICHT}

\section{Unfallmechanismen, die zu einem Thoraxtrauma} führen können

- Verkehrsunfall:

- Hochrasanztrauma PKW, LKW, KRad

- Kontakt mit Lenkrad

- als Fußgänger erfasst
> Tab. 1 Untersuchungsalgorithmus nach PHTLS ${ }^{\circledR}$ [2].

\begin{tabular}{|l|l|}
\hline ABCDE-Schema & Maßnahmen \\
\hline A - Airway-Management & $\begin{array}{l}\text { Inspektion der Atemwege, } \\
\text { Entfernen von Fremdkörpern/ } \\
\text { Erbrochenem, ggf. Guedel- } \\
\text { Tubus, manuelle HWS-Immo- } \\
\text { bilisation }\end{array}$ \\
\hline B - Breathing/Ventilation & $\begin{array}{l}\text { Atemfrequenz, Qualität der } \\
\text { Atemzüge, Inspektion der } \\
\text { Atemexkursionen, Auskulta- } \\
\text { tion, Palpation der Thoraxwand }\end{array}$ \\
\hline C - Circulation & $\begin{array}{l}\text { Suche nach Blutungsquellen } \\
\text { und Kontrolle (Verband), } \\
\text { Kontrolle Puls, Rekapillarisie- } \\
\text { rungszeit, } \text { SO }_{2} \text {, Blutdruck, } \\
\text { EKG }\end{array}$ \\
\hline D - Disability & $\begin{array}{l}\text { neurologische Untersuchung, } \\
\text { Detektion von Wirbelsäulen- } \\
\text { und Rückenmarksverletzungen }\end{array}$ \\
\hline $\begin{array}{l}\text { Entkleiden des Patienten, } \\
\text { orientierende Untersuchung, } \\
\text { Wärmeerhalt }\end{array}$ \\
\hline E - Exposure/Environment
\end{tabular}

- Insasse aus dem Fahrzeug geschleudert

- Insasse tot

- Sturz aus $\geq 3 \mathrm{~m}$ Höhe

- Druckwelle bei Explosion

\section{Diagnostik und klinische Untersuchung}

Die körperliche Untersuchung umfasst die klassische Inspektion mit besonderer Beachtung der Atemfrequenz und Dyspnoe. Mittels Auskultation werden die Atemgeräusche beurteilt: vorhanden, seitengleich, pathologische Geräusche? Eine Palpation soll Schmerzpunkte, Krepitationen, Hautemphyseme und Instabilitäten aufdecken. Es kann perkutiert werden. Ein hypersonorer Klopfschall weist auf einen Pneumothorax hin.

Ein strukturiertes Vorgehen ist bei der klinischen Untersuchung notwendig. Im Rahmen eines 5-SekundenChecks wird geprüft, ob eine Atemwegsverlegung, eine lebensbedrohliche Blutung oder ein traumatischer Herzstillstand vorliegt. Diese 3 Bedingungen führen zu einer Veränderung der Versorgungsprioritäten.

Wenn dies ausgeschlossen werden kann, wird strukturiert nach Untersuchungsalgorithmen von ERC oder $\operatorname{PHTLS}^{\circledR}$ ( $\triangleright$ Tab. 1) vorgegangen, um keine relevante Verletzung zu übersehen. 


\section{FALLBEISPIEL}

Ein 56-jähriger Mann fährt am frühen Morgen mit seinem PKW zur Arbeit. Beim vor ihm fahrenden LKW löst sich plötzlich der Auflieger, und es kommt zur Kollision, wobei sich der Auflieger mit der linken Seite des PKW verkeilt. Der Fahrer als einziger Insasse ist angeschnallt, die Airbags haben ausgelöst.

Der Patient ist eingeklemmt, schwer zugänglich und nur durch eine aufwendige technische Rettung zu befreien. Die initiale $\mathrm{S}_{p} \mathrm{O}_{2}$ zeigt $71 \%$, er hat eine Schnappatmung (AF 6/min) mit Zyanose und ist bewusstseinsgetrübt (GCS 3).

Die Rettungskräfte gelangen über die Beifahrertür zum Patienten. Über einen Larynxtubus wird der Patient assistiert beatmet, ein erster Venenzugang kann am rechten Arm etabliert werden. Darüber wird bei schlecht tastbarem Puls fraktioniert Adrenalin verabreicht. Der NEF-Fahrer hält Kontakt zum Einsatzleiter der Feuerwehr zwecks Absprache der Befreiung. Parallel wird eine Notfallnarkose mit trachealer Intubation vorbereitet.

\section{Stumpfes Thoraxtrauma}

\section{Lungenkontusion}

Die häufigste Folge des stumpfen Thoraxtraumas ist eine Lungenkontusion. Das Parenchym wird zerrissen, es kommt zur Einblutung in die Alveolen und zur Organschwellung. Die Lungenfunktion verschlechtert sich aufgrund verminderter Elastizität und einer Störung der Durchblutung. Es strömt vermehrt unzureichend oxygeniertes Blut in den Körperkreislauf. Eine Zyanose und eine Dyspnoe entwickeln sich.

\section{Merke}

Die wichtigste Erstmaßnahme am Unfallort ist die Sauerstoffgabe mit Maske und Reservoirbeutel.

Eine adäquate Analgesie soll die Schonatmung reduzieren und die Atemmechanik verbessern. Ist die Oxygenierung nicht ausreichend, kann bei ansprechbaren Patienten eine NIV-Therapie begonnen werden (CPAP, BiPAP). Bei polytraumatisierten Patienten müssen eine Sicherung der Atemwege und eine mechanische Beatmung erwogen oder zwingend durchgeführt werden. Kriterien sind:

- Apnoe oder Schnappatmung (Atemfrequenz <6),

- Hypoxie $\left(\mathrm{S}_{\mathrm{p}} \mathrm{O}_{2}<90 \%\right)$ trotz $\mathrm{O}_{2}$-Gabe und nach Ausschluss eines Spannungspneumothorax,

- schweres Schädel-Hirn-Trauma (GCS <9),

- traumaassoziierte hämodynamische Instabilität (systolischer Blutdruck < $90 \mathrm{mmHg}$ ),

- schweres Thoraxtrauma mit respiratorischer Insuffizienz (Atemfrequenz > 29/min) [3].
Merke

Dyspnoe und Zyanose sind Leitsymptome intrathorakaler Verletzungen. Rippenfrakturen müssen nicht zwingend vorliegen.

\section{Rippenfraktur/instabiler Thorax}

Brustschmerzen und Atemnot nach Unfall sind verdächtig auf eine Rippenfraktur. Bei Inspektion ist auf Gurt- oder Prellmarken zu achten. Auch weitere Begleitverletzungen sind Indikatoren. So sollte auf Klavikulafrakturen und Verletzungen der gesamten Wirbelsäule aufmerksam geachtet werden. Eine Rippenfraktur muss zwar nicht zwingend mit inneren Verletzungen einhergehen, aber Prellmarken und Frakturen sind Marker des Schweregrads der Verletzung [4]. Eine Fraktur der 1. Rippe, die präklinisch nicht eindeutig diagnostiziert werden kann, ist Hinweis auf eine hohe Energieeinwirkung und damit auch auf eine intrathorakale Gefäßverletzung [5].

Sind mehrere Rippen mehrfach gebrochen (mindestens 3 Rippen $2 \times$ gebrochen), wird der Brustkorb instabil („flail chest“). Es kommt zur paradoxen Atmung; der Patient hat eine schwere Dyspnoe und kann leicht dekompensieren bzw. ist respiratorisch insuffizient. Bei der Rippenserienfraktur liegt häufig eine Lungenkontusion vor; sie erfordert meist Intubation und Beatmung [6].

\section{Pneumothorax/Spannungspneumothorax}

Wird der Pleuraspalt von innen oder außen eröffnet, entsteht ein Pneumothorax. Beim stumpfen Thoraxtrauma dringen Rippen in das Lungengewebe ein und schaffen eine Verbindung vom Bronchialsystem zum Pleuraspalt. Es kann sich ein Hautemphysem entwickeln, die Auskultation ist abgeschwächt. Im Fall eines Ventilmechanismus dringt weitere Luft ein, kann nicht mehr entweichen, und Lunge, große Gefäße und Herz werden zunehmend komprimiert. Infolge einer Mediastinalverlagerung fällt der venöse Rückstrom ab, es kommt zur hämodynamischen Instabilität.

Cave

- Durch den Spannungspneumothorax ist der Patient vital bedroht (Erhöhung der intrathorakalen Drücke und konsekutiv Kreislaufstillstand). Eine sofortige Entlastung ist notwendig.

- Unter Beatmung kann sich aus einem einfachen Pneumothorax ein Spannungspneumothorax entwickeln.

- Auch der einfache Pneumothorax ist im Verlauf zu reevaluieren. 


\section{ÜBERSICHT}

Alarmsymptome

- zunehmende Atemnot bzw. erhöhte Beatmungsdrücke

- einseitig abgeschwächtes oder fehlendes Atemgeräusch

- Halsvenenstauung

- Schock, Kreislaufdekompensation, Tachykardie

\section{Behandlung}

Ein Spannungspneumothorax ist umgehend durch eine Dekompression zu behandeln. Die Nadeldekompression kann beim vital bedrohten Patienten durch Notfallsanitäter erfolgen. - Abb. 2 zeigt eine Handlungsanweisung.

\section{Merke}

Nach einer Nadeldekompression eines Spannungspneumothorax ist die Anlage einer Thoraxdrainage dringend empfohlen.

Die chirurgische Dekompression bzw. definitive Anlage einer Thoraxdrainage erfolgt durch den Notarzt. Sie wird regelhaft durch eine Minithorakotomie in Bülau-Position durchgeführt.

Die Präparation zum Pleuraspalt erfolgt stumpf, und die Drainage wird fingergeführt oder mittels Kornzange eingelegt. Keinesfalls wird eine mit Mandrin armierte Drainage verwendet, da dies das Verletzungsrisiko erheblich erhöht.

\section{Hämatothorax}

In etwa 1/3 der Fälle liegt beim schweren Thoraxtrauma ein Hämatothorax vor. Ursache ist meist eine Verletzung der Interkostalgefäße im Rahmen einer Rippenfraktur oder eine Verletzung durch penetrierende Einwirkung. Dies führt häufig zum Hämatopneumothorax. Klinisch imponieren das abgeschwächte Atemgeräusch und je nach Form eine typischerweise abgeschwächte Perkussion. Je nach Blutverlust entsteht ein hämorrhagischer Schock.

Primär ist eine Ateminsuffizienz zu beachten; bei Beatmung kann die Oxygenierung durch erhöhte intrathorakale Drücke erschwert sein. In diesem Fall ist ebenfalls eine Thoraxdrainage anzulegen und der Hämatothorax fraktioniert zu entlasten [2, 8]. Eine Nadeldekompression ist nicht zielführend.

\section{Verletzungen von Herz und Gefäßen}

Prellmarken über dem Sternum oder eine Sternumfraktur weisen auf eine mögliche Schädigung des Herzens hin. Schon geringe Traumata können die elektrische Aktivität beinträchtigen und zu bedrohlichen Arrhythmien führen. Als Folge einer Herzkontusion sind strukturelle Verletzungen von Herzgewebe oder Herzklappen möglich, die zu einem kardiogenen Schock führen können.

Wenn eine bestehende Hypotension durch andere Befunde (Hämatopneumothorax, andere Blutverluste) nicht plausibel erklärbar ist, muss an eine Perikardtamponade gedacht werden. Die Diagnose ist optimalerweise durch eine FAST-Sonografie zu stellen, die noch nicht flächendeckend präklinisch vorgehalten wird.

Therapie ist eine Entlastungspunktion von subxiphoidal. Kontrovers diskutiert werden Notfallthorakotomien (Clemmshell-Thorakotomie), die Expertise und Übung erfordern [9].

Bei großen Beschleunigungen oder Bremskräften kann es zu Scherbelastungen zwischen dem relativ freien Herzen und den fixierteren großen Gefäßen kommen mit möglicher Dissektion oder Ruptur der Aorta. Klinisch imponiert der resultierende Schock. Das Behandlungskonzept folgt dem anderer Gefäßnotfälle mit kontrollierter Hypotension. Eine spezifische Behandlung ist in der Präklinik nicht möglich.

\section{Penetrierendes Thoraxtrauma}

Das penetrierende Thoraxtrauma ist ein seltenes Verletzungsmuster und macht unter $10 \%$ aller Thoraxtraumata aus. Häufigste Ursachen sind Stichverletzungen, meist im Rahmen gewaltsamer Auseinandersetzungen, Pfählungsverletzungen durch Verkehrs- oder Arbeitsunfälle, seltener Schuss- oder Explosionsverletzungen.

Auch bei ihnen ist der Unfallmechanismus von besonderer Bedeutung, da scheinbar kleine Eintrittsverletzungen zu bedrohlichen inneren Gewebeschädigungen führen können und ein zunächst respiratorisch und hämodynamisch stabiler Patient schnell dekompensieren kann.

\section{Schussverletzungen}

Bei Schussverletzungen entsteht durch die enorme Projektilgeschwindigkeit bei Hochgeschwindigkeitsgeschossen mit teilweise mehr als $600 \mathrm{~m} / \mathrm{s}$ eine temporäre Wundhöhle, die ein Vielfaches der Größe der bleibenden Wundhöhle beträgt. Die kurzfristige, massive Verdrängung von Gewebe nach Eindringen des Projektils bedingt eine weitreichende Gewebeschädigung auch ohne direkte Geschossberührung noch fernab des eigentlichen Schusskanals [10]. Auch aus einer möglichen Austrittswunde lassen sich keine genauen Aussagen über den Verlauf des Schusskanals ableiten, da dieser nicht gerade durch den Körper verlaufen muss. 


\section{ABCDE-Herangehensweise + Notarztnachforderung}

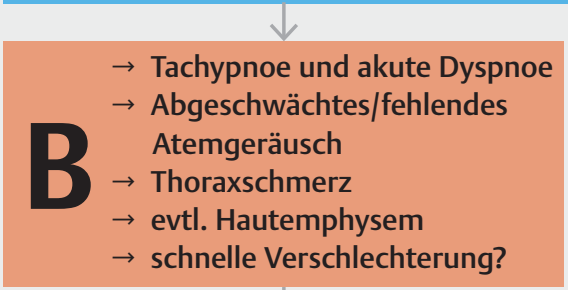

JA

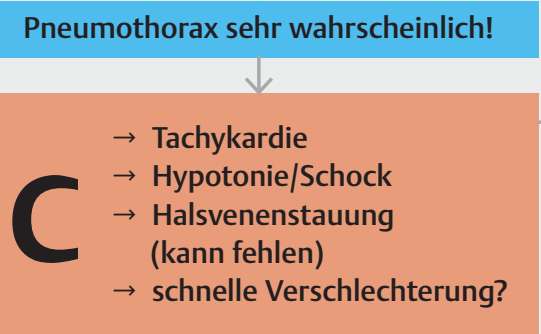

$\mathrm{JA}$

Pneumothorax anamnestisch und im

Gesamtkontext am wahrscheinlichsten?

\section{Spannungspneumothorax sehr wahrscheinlich!} $\downarrow$ JA

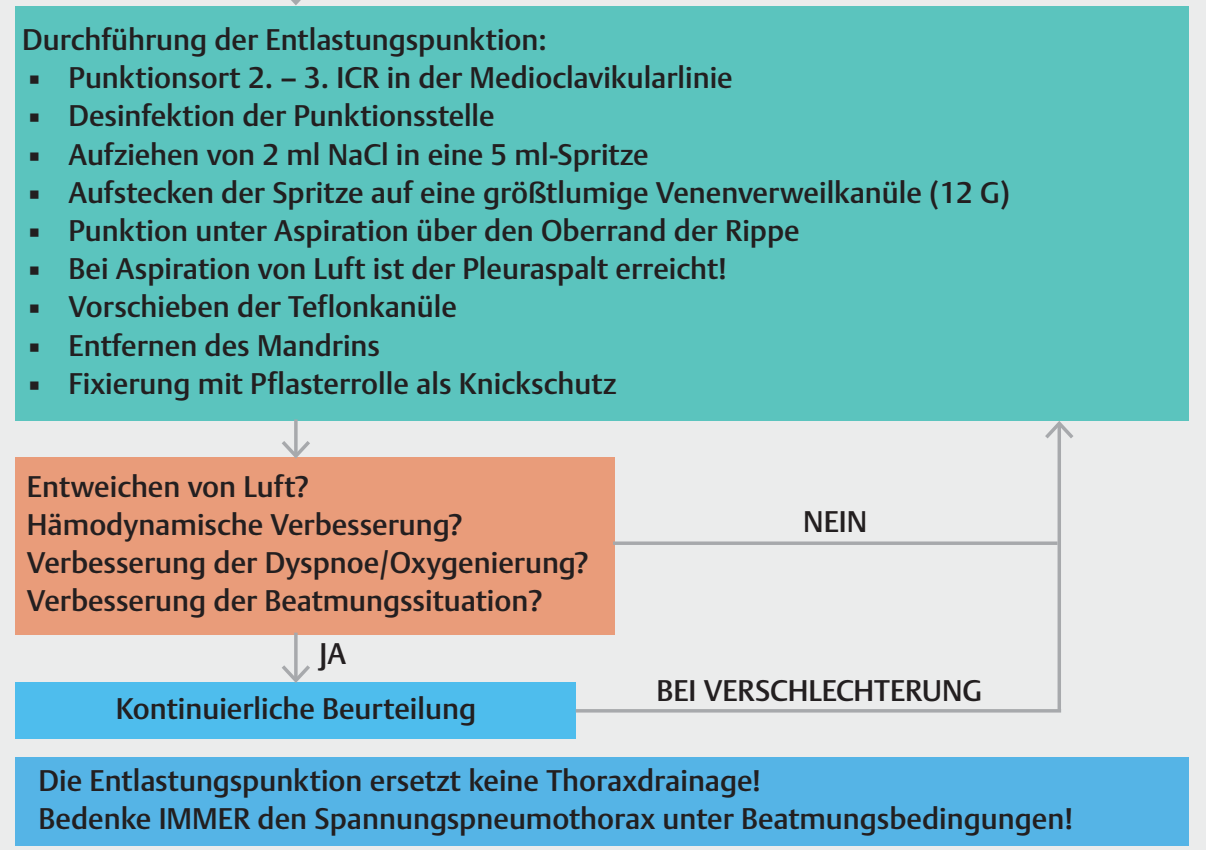

Abb.2 Indikation und technische Durchführung eine Entlastungspunktion bei Spannungspneumothorax (nach „Algorithmen für Notfallsanitäter DBRD“ [7]). 
In jedem Fall ist eine Schusswunde einer möglichst zügigen operativen Versorgung zuzuführen [1].

\section{Cave}

Auch bei kleinen Wunden können vital bedrohliche innere Gewebeschädigungen vorliegen.

\section{Pfählungs- und Stichverletzungen}

Pfählungs- und Stichverletzungen sind in Deutschland deutlich häufiger anzutreffen. Aufgrund des charakteristischen Verletzungsmechanismus lässt sich beim noch in der Wunde befindlichen Fremdkörper eine grobe Abschätzung möglicher innerer Verletzungen treffen. Ein Fremdköper darf präklinisch keinesfalls aus der Wunde entfernt werden und sollte lediglich unter möglichst sterilen Bedingungen gegen Verrutschen und weiteres Eindringen gesichert werden, um eine Ausdehnung der inneren Verletzung durch Bewegung zu verhindern. Zudem tamponiert der Fremdkörper möglicherweise die Blutungsquelle.

\section{Cave \\ Noch in der Wunde befindliche Fremdkörper dürfen nur operativ entfernt werden. Nach Stabilisierung des Patienten sind Fremdkörper gegen Verrutschen zu sichern.}

Ist bei einer Stichverletzung der Fremdkörper bereits aus der Wunde entfernt, wird die Wunde evtl. übersehen oder das Ausmaß der dahinterliegenden Verletzung unterschätzt.

\section{PRAXIS}

\section{Begleitverletzungen}

Bei penetrierenden Verletzungen unterhalb der Mammillarlinie ist zusätzlich von einem begleitenden Abdominaltrauma auszugehen. Somit kann es wie beim stumpfen Thoraxtrauma zu Verletzungen aller Thoraxorgane und der oberen abdominalen Organe (Leber, Milz, Pankreas, Magen, obere Darmabschnitte und Zwerchfell) kommen.

In der Mehrheit der Fälle zielen thorakale Stichverletzungen in Richtung des Herzens, treffen jedoch häufiger die A. thoracica interna oder die Lunge [11]. Dabei können infolge veränderter Lagebeziehungen in In- und Exspiration Verschiebungen von bis zu $10 \mathrm{~cm}$ im Verlauf des Stichkanals entstehen [12]. Bei einer luftsaugenden Wunde ist eine besondere Behandlung notwendig, da ein sich bildender Ventilmechanismus einen Spannungspneumothorax verursachen kann. Diese Wunden können z. B. mit dem Asherman Chest Seal ${ }^{\circledR}$ behandelt werden. Alternativ kann eine luftdichte, sterile Auflage auf die Wunde aufgebracht werden, die an einer Seite nicht verklebt wird, damit Luft entweichen kann. Gegebenenfalls wird präklinisch eine Thoraxdrainage angelegt [3].

\section{Weiteres Vorgehen}

Die weitere Behandlung ist zielgerichtet je nach Atemund Kreislaufinsuffizienz. Sicherung der Atemwege, Intubation und Volumengabe stehen im Vordergrund. Über 2 großlumige Zugänge wird die Volumentherapie mittels kristalloider Infusionslösung mit dem Ziel einer permissiven Hypotonie begonnen.

Eine Narkose kann entsprechend den Richtlinien der DGAI [13] ausgeführt werden. Bei kreislaufinstabilen Patienten ist Ketamin vorzuziehen, da mit weiterer Kreislaufdepression gerechnet werden muss.

Die präklinische Zeit ist sehr kurz zu halten, da dies das Outcome beeinflusst [14] und eine definitive Versorgung nur innerklinisch möglich ist und die Zielklinik abhängig vom Verletzungsmuster gewählt werden sollte. Die mittlere präklinische Zeit beim Polytrauma im Traumaregister der DGU 2017 könnte mit 66 Minuten für diese Patienten zu lang sein [15].

\section{Merke \\ Jeder Zeitverlust in der Behandlungssituation vor Ort ist somit zu vermeiden.}

\section{FALLBEISPIEL}

Nach 20 Minuten kann der Patient aus dem Fahrzeug gerettet werden. Der Blutdruck liegt bei $80 \mathrm{mmHg}$ systolisch, die linke Körperhälfte zeigt schwere Verletzungen: am Kopf eine blutige Rissquetschwunde, am Thorax links Krepitationen, ein Weichteilemphysem und abgeschwächte Atemgeräusche sowie eine Klavikulafraktur. Der linke Arm steht grotesk ab mit Frakturen am Ober- und Unterarm. Am linken Knie sind offene, blutende Gelenkfrakturen zu erkennen. Nach Notfallnarkose mit Sicherung des Atemwegs punktiert die Notärztin den Thorax $2 \times$ medioklavikulär links zur Entlastung des Spannungszustands. 2 weitere periphere Zugänge können etabliert werden. Anschließend erfolgt der Transport unter Katecholaminpflicht in das $25 \mathrm{~km}$ entfernte Traumazentrum. Ein RTH steht zu diesem Zeitpunkt nicht zur Verfügung.

Im Schockraum wird nach der Thoraxröntgenaufnahme bei Hämatopneumothorax, Rippenserien- und Sternumfraktur eine Drainage in Bülau-Position eingelegt. 500 ml Blut entleeren sich. Nach der CT-Diagnostik kann der weiter instabile und diffus blutende Patient einer Damage Control Surgery mit Anlage mehrerer Fixateurs externes an den linken Extremitäten zugeführt werden. 
Bei bestehendem Schädel-Hirn-Trauma werden erhöhte Hirndruckwerte gemessen. Nach einer verlängerten Intensivtherapie kann der Patient mit erheblichen zerebralen Einschränkungen in die Frührehabilitation verlegt werden.

\section{Transport}

Bei der Auswahl einer geeigneten Klinik ist die weiterführende Diagnostik und Therapie entscheidend. Patienten mit schweren Verletzungen und gestörten Vitalfunktionen sollten in ein überregionales Traumazentrum transportiert werden. Zur Überbrückung längerer Strecken ist die Luftrettung von Vorteil. Eine Reevaluation hat vor Transportbeginn zu erfolgen. Aufgrund der Flugphysiologie und des eingeschränkten Zugangs zum Patienten wird die Intubation niederschwelliger indiziert.

\section{Schlussfolgerung}

Grundsätzlich können Patienten mit thorakaler Verletzung in 4 chirurgische Kategorien eingeteilt werden [15]:

- Patienten, die durch Anlage einer Thoraxdrainage suffizient versorgt sind ( $80-85 \%$ aller Fälle mit Thoraxtrauma).

- Patienten, die entweder unmittelbar vor Ort oder im Schockraum/OP versterben (Verletzungen des Herzens oder der großen Gefäße).

- Patienten mit potenziell lebensbedrohlichen Verletzungen, die nach initialer Stabilisierung dringlich diagnostiziert und operativ versorgt werden müssen (z. B. Blutungen aus Interkostalarterien oder dem Lungenparenchym).

- Patienten, die nach initialer Drainagetherapie noch Residuen (anhaltende Blutung, chronischer Hämato-/ Pneumothorax, Empyem, instabiler Thorax etc.) aufweisen und sekundär operativ versorgt werden müssen (15-20\% der Patienten aus der erstgenannten Gruppe).

\section{KERNAUSSAGEN}

- Im deutschsprachigen Raum dominieren stumpfe Thoraxtraumata. Penetrierende Verletzungen treten häufiger in Großstädten und in suizidaler Absicht auf.

- Die Beurteilung umfasst den Unfallmechanismus und lässt von der einwirkenden Kinetik auf die Schwere eines Traumas schließen.

- Lungenkontusionen sind die häufigste Pathologie.

- Eine NIV-Beatmung kann erwogen werden. Häufig wird eine Intubation mit lungenprotektiver Beatmung unumgänglich.
- Nach Nadeldekompression eines Spannungspneumothorax ist die Anlage einer Thoraxdrainage zu empfehlen.

- Bei penetrierender Verletzung kann die Größe des inneren Traumas deutlich vom Ausmaß der äußeren Wunde abweichen.

- Hämodynamisch instabile Patienten sollten mit kurzer Prähospitalzeit behandelt werden.

- Transportmittel und Zielklinik sollten adäquat gewählt werden.

\section{Interessenkonflikt}

Die Autoren geben an, dass kein Interessenkonflikt besteht.

\section{Autorinnen/Autoren}

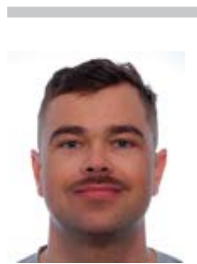

\section{Timo Keinert}

Dr. med., Zusastzbezeichnung Notfallmedizin Facharzt für Anästhesie, leitender Notarzt. Tätig an der Klinik für Anästhesie und operative Intensivmedizin, Katharinenhospital, Klinikum Stuttgart.

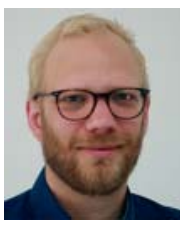

\section{Tobias Frech}

Notfallsanitäter und Dozent am mobile medic Lehrinstitut für Notfallmedizin, AHA-Instruktor. Aktiv im Rettungsdienst Stuttgart.

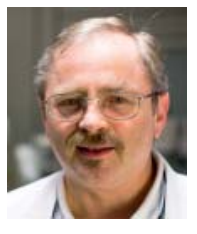

\section{Albrecht Henn-Beilharz}

Dr. med., Facharzt für Anästhesie, Zusatzbezeichnung Notfallmedizin und spezielle Intensivmedizin, Fachkunde Leitender Notarzt, ERCInstruktor. Tätig als Leitender Oberarzt der Klinik für Anästhesie und operative Intensivmedizin, Katharinenhospital, Klinikum Stuttgart.

\section{Korrespondenzadresse}

\section{Dr. med. Albrecht Henn-Beilharz}

Klinik für Anästhesie und operative Intensivmedizin

Klinikum Stuttgart - Katharinenhospital

Kriegsbergstraße 60

70174 Stuttgart

a.henn-beilharz@klinikum-stuttgart.de

\section{Literatur}

[1] Schulz-Drost S, Matthes G, Ekkernkamp A. Erstversorgung des Patienten mit schwerem Thoraxtrauma. Notfall Rettungsmed 2015; 18 (5): $421-437$

[2] Hansen M, Hachenberg T. Der Patient mit Thoraxtrauma: präklinische Versorgung. Anästhesiol Intensivmed Notfallmed Schmerzther 2017; 52: 408-421

[3] Deutsche Gesellschaft für Unfallchirurgie - DGU. S3-Leitlinie Polytrauma. AWMF-Registernummer 012 - 019. Im Internet: 
https://www.awmf.org/leitlinien/detail/II/012-019.html Stand: 07.07.2018

[4] Horst K, Hildebrand F, Kobbe P et al. Detecting severe injuries of the upper body in multiple trauma patients. J Surg Res 2015; 199 (2): 629-634. doi: 10.1016/j.jss.2015.06.030. 2015

[5] Murphy CE, Raja AS, Baumann BM et al. Rib fracture diagnosis in the panscan era. Ann Emerg Med 2017; 70 (6): 904 - 909

[6] Simon B, Ebert ], Bokhari F et al. Management of pulmonary contusion and flail chest. J Trauma 2012; 73 (5): S351 - S361

[7] Deutscher Berufsverband Rettungsdienst - DBRD. MusterAlgorithmen zur Umsetzung des Pyramidenprozesses im Rahmen des NotSanG, Version 2.0. Im Internet: http://dbrd.de/ images/algorithmen/AlgorithmenNotSanDBRD2017V2.0.pdf Stand: 07.07.2018

[8] Bölükbas S, Ghezel-Ahmadi AK, Kwozalla JS. Diagnostik und Behandlungskonzepte beim Thoraxtrauma. Chirurg 2011; 82: 843

[9] Nevins EJ, Moori PL, Smith-Williams J et al. Should pre-hospital resuscitative thoracotomy be reserved only for penetrating chest trauma? Eur J Trauma Emerg Surg 2018. (Epub ahead of print)

[10] Khodadadyan C, Hoffmann R, Neumann K et al. The diagnostic value of thoracic computerized tomography in severe thoracic trauma. Chirurg 1995; 66: $1097-1103$
[11] Wollf M, Hirner A. Thoraxverletzungen. In: Weise K, Hirner A, Hrsg Chirurgie. Stuttgart: Thieme-Verlag; 2008

[12] Eggeling S, Passlick B. Thoraxverletzungen. In: Wirth C], Mutschler W, Hrsg Praxis der Orthopädie und Unfallchirurgie. Stuttgart: Thieme-Verlag; 2014

[13] Deutsche Gesellschaft für Anästhesiologie und Intensivmedizin - DGAI. Handlungsempfehlung: Prähospitale Notfallnarkose beim Erwachsenen. Anästh Intensivmed 2015; 56: $317-335$

[14] Harmsen AM, Giannakopoulos GF, Moerbeek PR et al. The influence of prehospital time on trauma patients outcome: a systematic review. Injury 2015; 46: 602 -609

[15] Deutsche Gesellschaft für Unfallchirurgie - DGU. Traumaregister der DGU. de/TR-DGU-Jahresbericht_2017.pdf

[16] Ahmed N, Jones D. Video assisted thoracic surgery: state of the art in trauma care. Injury 2004; 35 (5): 479-489

Bibliografie

DOI https://doi.org/10.1055/s-0043-118699 retten 2018; 7: 276-285

(c) Georg Thieme Verlag KG, Stuttgart · New York ISSN 2193-2387 
Punkte sammeln auf CEEthiemede

Diese Fortbildungseinheit ist 12 Monate online für die Teilnahme verfügbar.

Sollten Sie Fragen zur Online-Teilnahme haben, finden Sie unter cme.thieme.de/hilfe

eine ausführliche Anleitung. Wir wünschen viel Erfolg beim Beantworten

der Fragen!

Unter https://eref.thieme.de/ZZX92KV oder über den QR-Code kommen Sie direkt zum Artikel zur Eingabe der Antworten.

\section{Frage 1}

Welche Aussage zur Epidemiologie des Thoraxtrauma ist falsch?

A Das stumpfe Thoraxtrauma überwiegt.

B Das penetrierende Trauma ist in Westeuropa sehr häufig.

C Ein Thoraxtrauma betrifft alle Altersstufen bis zu immer häufiger geriatrischen Patienten.

D Verletzungen des Brustkorbs finden sich häufig beim Polytrauma.

E Die präklinische Mortalität hängt von der erfolgreichen Behandlung eines Thoraxtraumas ab.

\section{Frage 2}

Was sollte bei der Ersteinschätzung eines Thoraxtraumas bedacht werden?

A Der sog. 5-Sekunden-Check umfasst die Prüfung, ob eine Atemwegsverlegung, eine lebensbedrohliche Blutung oder ein traumatischer Herzstillstand vorliegt.

$B$ Die Messung der $\mathrm{S}_{\mathrm{p}} \mathrm{O}_{2}$ reicht aus. So übersieht man keine Verletzung.

C Klavikulafrakturen stehen nicht im Zusammenhang mit Traumata des Brustkorbs.

D Prellmarken sind nur bei Antikoagulierten von Bedeutung.

E Das instabile Thoraxtrauma ist ein symptomarmes, diagnostisches „Chamäleon“.

\section{Frage 3}

Einige Verletzungen bedürfen einer zügigen präklinischen Behandlung. Welche Aussage ist richtig?

A Die Beurteilung von Atemfrequenz und der Qualität der Atemzüge, die Inspektion der Atemexkursionen sowie die Auskultation sind dem Airway-Management im ABCDE-Schema zugeordnet.

B Verlegungen der Atemwege stehen nie im Vordergrund.

C Lungenkontusionen werden in der Präklinik primär mit Sauerstoff behandelt.

D Perikarddrainagen gehören zum Standardrepertoire von Notärzten.

E Patienten mit Schmerzen haben i.d.R. keine gewichtige Verletzung des Thorax.

\section{Frage 4}

Einer der folgenden Befunde gehört nicht zu den Leitsymptomen des Spannungspneumothorax. Welcher?

A Hypotonie/Kreislaufdekompensation

B abgeschwächte Atemgeräusche

C Tachykardie

D Schmerzen

E gestaute Halsvenen

\section{Frage 5}

Eine Entlastungspunktion therapiert einen Spannungspneumothorax. Welche Aussage in diesem Zusammenhang ist richtig?

A Sie ist zwingend notärztliche Maßnahme.

B Unter maschineller Beatmung braucht es keine Entlastungspunktion.

C Die Entlastungspunktion wird im 4./5. Interkostalraum durchgeführt.

D Nach Punktion ist ein Pneumothorax dauerhaft entlastet.

E Die Fehleinschätzung eines Spannungspneumothorax kann die intrathorakalen Drücke deutlich erhöhen und führt zum Kreislaufstillstand.

\section{Frage 6}

Eine der folgenden Aussagen zum Transport von Verletzten mit Thoraxtrauma ist falsch. Welche?

A Die initiale Symptomatik und der klinische Befund sollten dynamisch betrachtet werden.

B Beim luftgebundenen Transport sollte eine invasive Beatmung möglichst vermieden werden.

C Schwer Polytraumatisierte erfordern präklinisch im Einzelfall besondere Maßnahmen (z. B. Intubation, Drainagenanlage).

D Häufig werden Blutverluste unterschätzt, v. a. beim stumpfen Thoraxtrauma. Die Notfallnarkose kann eine Hypovolämie demarkieren.

E Indikationen zur Intubation sind z. B. deutliche Hypoxie und hämodynamische Instabilität.

- Weitere Fragen auf der folgenden Seite... 


\section{Punkte sammeln auf CEE.thieme.de}

\section{Frage 7}

Wodurch ist der Pneumothorax gekennzeichnet?

A Jeder Pneumothorax erfordert eine sofortige Thoraxdrainagenanlage.

B Bei offenem Pneumothorax ist eine Drainage nie notwendig.

C Ein einfacher Pneumothorax sollte im Verlauf reevaluiert werden.

D Bei Beatmung mit hoher $\mathrm{F}_{\mathrm{i}} \mathrm{O}_{2}$ ist eine Punktion nicht notwendig.

E Entlastungspunktionen sind in Deutschland ausschließlich in Bülau-Position zulässig.

\section{Frage 8}

Die Volumentherapie ist oft „Stiefkind“ der präklinischen Versorgung. Welche Aussage in diesem Zusammenhang ist falsch?

A Hämodynamische Instabilität kann von einem Hämatothorax verursacht werden.

B Intraossäre Zugänge dienen neben peripheren Zugängen auch präklinisch zur Volumentherapie polytraumatisierter Patienten.

C Bei einem Blutverlust kann ein Volumenbolus von $20 \mathrm{ml} / \mathrm{kg} \mathrm{KG}$ Vollelektrolytlösung sinnvoll sein.

D Eine übermäßige Infusionstherapie kann zur Akzeleration von Schädigungen, v. a. Lungenkontusionen, führen.

E Kolloidale Lösungen sind die Therapie der 1. Wahl bei Schwerverletzten mit Thoraxtrauma.

\section{Frage 9}

Was trifft auf einen sog. instabilen Thorax zu?

A Beim instabilen Thorax müssen immer eine Intubation und eine mechanische Beatmung durchgeführt werden.

B Häufig ist der instabile Thorax bei Schuss- oder Stichverletzungen anzutreffen.

C Ein Thoraxwandsegment wird ab 5 Rippenpaaren à 2 Frakturen instabil.

D Ein instabiler Thorax geht praktisch nie mit Lungenkontusionen einher.

E Klinisches Symptom sind paradoxe Atembewegungen.

\section{Frage 10}

Nur eine der folgenden Aussagen zum penetrierenden Thoraxtrauma ist korrekt. Welche?

A Bei Pfählungen sind die Fremdkörper kontaminiert und müssen präklinisch entfernt werden. Ein Chest Seal dient zur Abdeckung.

B In deutschen Großstädten sind $50 \%$ aller Traumata des Thorax penetrierend.

C Das Prinzip „Load and go" steht immer im Vordergrund. Es braucht keinen peripheren Zugang.

D Ein Stichkanal erlaubt eine vollständige Beurteilung der Verletzung.

E Die Wahl der Zielklinik ist abhängig vom Verletzungsmuster. 